Pacific Journal of Mathematics

ENTROPY AND SINGULARITY OF INFINITE CONVOLUTIONS 


\title{
ENTROPY AND SINGULARITY OF INFINITE CONVOLUTIONS
}

\author{
Adriano M. Garsia
}

Introduction. Let $\Phi(\omega)$ be a random variable which takes only a finite number of values

$$
x_{1}, x_{2} \cdots, x_{p},
$$

with probabilities

$$
\pi_{1}, \pi_{2}, \cdots, \pi_{p}
$$

Let $A(x)$ be the distribution function of $\Phi(\omega)$.

We shall be concerned here with infinite convolutions of the type

$$
F(x, r)=A\left(\frac{x}{r_{1}}\right) * A\left(\frac{x}{r_{2}}\right) * \cdots * A\left(\frac{x}{r_{n}}\right) * \cdots
$$

where $r=\left(r_{1}, r_{2}, \cdots, r_{n}, \cdots\right)$ is a given sequence of non-vanishing real numbers. From standard theorems of Probability theory it follows that the convolution product in (I.1) converges (if we exclude the trivial case $p=1, x_{1}=0$ ) if and only if $\sum r_{n}^{2}<\infty$ and either

$$
E(\Phi)=0
$$

or

$$
E(\Phi) \neq 0 \text { but } \sum r_{n} \text { is convergent. }
$$

In either case, the limit distribution $F(x, r)$ is continuous and pure. ${ }^{1}$ A proof of this result in the case that $\Phi(\omega)$ takes only the two values \pm 1 with equal probabilities can be found in [4].

We can and shall restrict our study to the case $E(\Phi)=0$. Our main result here concerns the distributions $F(x, r)$ generated by sequence $\left\{r_{n}\right\}$ such that, for some $0<\beta<1$,

$$
r_{n}=0\left[\beta^{n}\right] \text {. }
$$

Under this hypothesis it is easy to see that for a given $A(x)$, when $\beta$ is sufficiently small, $F(x, r)$ is necessarily singular. This result follows from the simple fact that the set of points of increase of $F(x, r)$, for all sufficiently small $\beta$, has zero measure. On the other hand, as $\beta$ increases towards one $F(x, r)$ in general will become

Received January 30, 1963. This work was carried out while the author was supported by the A. F. Office of Scientific Research.

1 That is either absolutely continuous or purely singular. 
absolutely continuous. We are interested in finding a lower bound for the $\beta$ 's for which this may happen. Considerations involving the set of points of increase of $F(x, r)$ stop short of being helpful for, as we shall see, in general we can be sure that $F(x, r)$ will remain singular beyond the first $\beta$ for which this set acquires positive measure.

Our discovery consists in the fact that, without further information on the relations between the values $x_{1}, x_{2}, \cdots, x_{p}$ and the sequence $\left\{r_{n}\right\}$, the best possible dividing line between singularity and absolute continuity of $F(x, r)$ is given by the entropy [8] of the distribution $\Phi(\omega)$.

More specifically we shall show that

THEOREM I.1 If $\left\{\boldsymbol{r}_{n}\right\}$ satisfies I.4 and

$$
-\sum_{i=1}^{p} \pi_{i} \log \pi_{i}<\log 1 / \beta,
$$

then the function $F(x, r)$ is necessarily singular.

Our methods bring also to light some peculiarities of the PisotVijayaraghavan numbers. The latter are algebraic integers whose conjugates are all in absolute value less than one [6]. Let $\alpha$ be in the interval $(1,2)$ and set $\beta=1 / \alpha$. Let $H_{p}(\alpha)$ denote the entropy of the distribution of the random variable

$$
y_{p}=\varepsilon_{1} \beta+\varepsilon_{2} \beta^{2}+\cdots+\varepsilon_{p} \beta^{p},
$$

where the $\varepsilon_{i}$ are independent random variables taking the values \pm 1 with equal probabilities. We can show the following result:

TheOREM I.2. For every $\alpha$ in $(1,2)$ the ratio $H_{p}(\alpha) / p$ is convergent, and if $\alpha$ is a P.V. number

$$
\lim _{p \rightarrow \infty} H_{p}(\alpha) / p<\log \alpha
$$

We note that Theorems I.1 and I.2 combined provide an explanation for the singularity [2] of the distribution function of the random variable

$$
y=\sum_{n=1}^{\infty} \varepsilon_{n} \beta^{n},
$$

when $\beta$ is the reciprocal of a P. V. number ${ }^{2}$. This is obtained by letting $A(x)$ be the distribution of the variable $y_{p}$ and setting

\footnotetext{
${ }^{2}$ Cfr. [3] for further references and a history of this question.
} 
$r_{n}=\beta^{n p}$, then taking $p$ sufficiently large.

The inequality in (I.6) contains the fact that the numbers

$$
\pm \beta \pm \beta^{2} \pm \cdots \pm \beta^{p}
$$

for sufficiently large $p$, cannot be all distinct. This implies that each $\mathrm{P}$. V. number in $(1,2)$ satisfies a polynomial equation with coefficients \pm 1 or 0 . Two different proofs of this results can be found in [7] and [3]. Here we shall show that even more is true, namely

Theorem I.3 Let $1 / \beta$ be a $P$. $V$. number in the interval $(1,2)$, For any increasing sequence of integers $\left\{n_{k}\right\}$ such that

$$
(1 / \beta) n_{k}=0\left(2^{k}\right)
$$

the corresponding powers of $\beta$

$$
\beta^{n_{1}}, \beta^{n_{2}}, \cdots, \beta^{n_{k}}, \cdots
$$

cannot be independent over the coefficients $\pm 1,0$.

A result of the same type as Theorem I.1 holds for convolutions of the form

$$
F(x)=A_{1}(x) * A_{2}(x) * \cdots * A_{n}(x) * \cdots,
$$

where for each $n, A_{n}(x)$ is the distribution of a random variable which takes only a finite number of values. Such a function when it is defined, is either totally discontinuous, continuous but purely singular or absolutely continuous. We shall be concerned with the cases in which $F(x)$ is continuous.

We can visualize (I.7) as being the distribution of a sum of independent random variables

$$
y=x_{1}+x_{2}+\cdots+x_{n}+\cdots
$$

where $x_{n}$ has distribution $A_{n}(x)$. The continuity of $F(x)$ is assured as soon as $y$ is not probabilistically equivalent to a series of constants [5].

In the case that

$$
E\left(x_{n}\right)=0, \quad \sum E\left(x_{n}^{2}\right)<\infty
$$

the result corresponding to Theorem I.1 reads as follows. Let $H_{n}$ denote the entropy of the distribution $A_{n}(x)$.

THEOREM I.4. If $\left\{R_{n}\right\}$ is a sequence of positive numbers tending to zero for which

$$
\lim \inf R_{n}^{2} /\left(E\left(x_{n+1}^{2}\right)+E\left(x_{n+2}^{2}\right)+\cdots\right)>0
$$


then an estimate such as

$$
\lim \inf \left(H_{1}+H_{2}+\cdots+H_{n}\right) / \log 1 / R_{n}<1
$$

implies the singularity of $F(x)$.

It can be shown that, although the condition (I.9) is best possible, no estimates on the entropy of the partial sums

$$
y_{n}=x_{1}+x_{2}+\cdots x_{n}
$$

are necessary for the singularity of $F(x)$. However, it is worthwhile to note that the situation is quite different if we look for necessary and sufficient conditions for $F$ to be absolutely continuous. with a derivative in the $L \log L$ class. To this effect we have the following theorem. Let $y=y_{n}+z_{n}$ with $y_{n}$ and $z_{n}$ independent and bounded and

$$
E\left(z_{n}^{2}\right) \rightarrow 0 \quad \text { as } n \rightarrow \infty .
$$

Let $F(x)$ be the distribution of $y$ and $F_{n}(x)$ be that of $y_{n}$. Suppose: that $F(x)$ is continuous.

THEOREM I.5. If $A_{n}$ is a sequence of positive numbers tending. to zero and such that

$$
\lim \inf A_{n}^{2} / E\left(z_{n}^{2}\right)>0
$$

then a necessary and sufficient condition for $F$ to be absolutely continuous and $F^{\prime}$ to be in the $L \log L$ class is that as $n \rightarrow \infty$ we: have

$$
\begin{gathered}
\log 1 / A_{n}+\sum\left[F_{n}\left(k A_{n}+A_{n}\right)-F_{n}\left(k A_{n}\right)\right] \\
\cdot \log \left[F_{n}\left(k A_{n}+A_{n}\right)-F_{n}\left(k A_{n}\right)\right]=0(1) .
\end{gathered}
$$

\section{Auxiliary lemmas and definitions.}

1.1 It will be useful to consider sequences $\left\{\mathfrak{P}_{n}\right\}$ of ordered pro--. bability distributions

$$
\mathfrak{P}_{n}=\left(p_{1}(n), p_{2}(n), \cdots, p_{N(n)}(n)\right) .^{3}
$$

To be specific, for each $n$ we shall suppose that

(a) $p_{1}(n) \geqq p_{2}(n) \geqq \cdots \geqq p_{N(n)}(n) \geqq 0$

(b) $\quad p_{1}(n)+p_{2}(n)+\cdots+p_{N(n)}(n)=1$.

For a given probability distribution $\mathfrak{F}=\left(p_{1}, p_{2}, \cdots, p_{N}\right)$ the distribu-..

\footnotetext{
3 We shall assume that $N(n) \rightarrow \infty$ as $n \rightarrow \infty$.
} 
tion function $F(x)$ defined by the condition that

$$
F^{\prime}(x)=\left\{\begin{array}{l}
0 \text { for } x<0 \text { or } x \geqq 1 \\
N p_{i} \text { for }(i-1) / N \leqq x<i / N,
\end{array}\right.
$$

will be called the "associated" distribution function.

Given a sequence $\left\{\mathfrak{P}_{n}\right\}$ such that (1.11) and (1.12) hold we shall have the sequence $\left\{F_{n}\right\}$ of associated distribution functions and the family $\overline{\left\{F_{n}\right\}}$ of the distributions that can be obtained as limits of the $F_{n}$ 's.

1.2. Lemma 1.21. For a given $\left\{\Re_{n}\right\}$ the functions of $\left\{\overline{F_{n}}\right\}$, except for a possible jump at the origin, are absolutely continuous.

Proof. Because of (a) we have that for each $i=1,2, \cdots, N$.

$$
1 \geqq p_{1}+p_{2}+\cdots p_{i} \geqq i p_{i} ;
$$

this gives that for $(i-1) / N \leqq x<i / N$.

$$
F^{\prime}(x) \leqq N / i<1 / x \text {. }
$$

Thus the associated distribution functions are uniformly absolutely continuous on the right of one, hence the same will hold for their limits.

LEMma 1.22. The functions $F_{n}$ are uniformly absolutely continuous if and only if the functions of $\left\{\overline{F_{n}}\right\}$ are continuous at the origin.

Proof. First of all it is clear that the functions $F_{n}$ are uniformly absolutely continuous if and only if they are equicontinuous. But if the $F_{n}$ 's are equicontinuous, their limits are continuous. Vice versa if their limits are continuous at the origin, a standard argument shows that the $F_{n}$ 's must be equicontinuous.

Definition. If in the family $\left\{\overline{F_{n}}\right\}$. there are discontinuous functions, we shall say that $\left\{\mathfrak{P}_{n}\right\}$ is a "singular" sequence. In this case the maximum of the jumps of the functions of $\left\{\overline{F_{n}}\right\}$ will be called the "deficiency" of the family $\left\{\mathfrak{P}_{n}\right\}$.

Clearly $\left\{\mathfrak{P}_{n}\right\}$ has deficiency $\geqq \gamma>0$ if and only if there exists a subsequence $\left\{n_{k}\right\}$ such that for any $\varepsilon>0$ we have

$$
\lim _{k \rightarrow \infty}\left[p_{[\varepsilon N]}\left(n_{k}\right)+\cdots+p_{N}\left(n_{k}\right)\right] \leqq 1-\gamma \cdot \cdot^{4}
$$

\footnotetext{
" $[\varepsilon N]$ is to mean "integral part of $\varepsilon n$ ".
} 
1.3. We shall now give a sufficient condition for a $\left\{\mathfrak{P}_{n}\right\}$ to be a singular sequence.

LEMMA 1.3. The sequence $\left\{\mathfrak{P}_{n}\right\}$ fails to be singular only if

$$
\lim _{n \rightarrow \infty}-\left(\sum p_{i}(n) \log p_{i}(n)\right) / \log N(n)=1 .
$$

As a matter of fact, if (1.31) does not hold, the deficiency $\gamma$ of $\left\{\mathfrak{P}_{n}\right\}$ satisfies the inequality

$$
1-\gamma \leqq \liminf _{n \rightarrow \infty}-\left(\sum p_{i}(n) \log p_{i}(n)\right) / \log N(n) .
$$

Proof. Since a sequence $\left\{\mathfrak{P}_{n}\right\}$ is singular with deficience $\geqq \gamma$ if a. a subsequence $\left\{\mathfrak{P}_{n_{k}}\right\}$ is such, we can assume that

$$
\lim _{n \rightarrow \infty}-\left(\sum p_{i}(n) \log p_{i}(n)\right) / \log N(n)=1-\gamma_{0}<1 .
$$

We define the quantities $a_{k}\left(=a_{k}(n)\right)$ for $k=1,2, \cdots, N(n)$ by setting

$$
a_{k}=k\left[p_{k}-p_{k+1}\right] \quad\left(\text { set } p_{N+1}=0\right) \text {. }
$$

Note that from our assumption 1.12 (a) it follows that $a_{k} \geqq 0$. We: also have that

$$
p_{k}=a_{k} / k+a_{k+1} /(k+1)+\cdots+a_{N} / N \quad(k=1,2, \cdots, N) .
$$

From 1.12 (b) we have

$$
a_{1}+a_{2}+\cdots+a_{N}=p_{1}+p_{2}+\cdots+p_{N}=1
$$

From the concavity of $Y(x)=-x \log x$, for any nonnegative constants $\left(b_{1}, b_{2}, \cdots, b_{N}\right)$ we have

$$
\sum a_{k} Y\left(b_{k}\right) \leqq Y\left(\sum a_{k} b_{k}\right)
$$

Using this inequality with $\left(b_{1}, b_{2}, \cdots, b_{N}\right)$ replaced by

$$
\left(0,0, \cdots, 0, \frac{1}{i}, \frac{1}{i+1}, \cdots, \frac{1}{N}\right)
$$

we get

$$
-\sum_{k=i}^{N} a_{k} / k \log 1 / k \leqq-\left(\sum_{k=i}^{N} a_{k} / k\right) \log \left(\sum_{k=i}^{N} a_{k} / k\right)=-p_{i\rfloor}{ }^{\prime} \log p_{i} .
$$

Summing with respect to $i$ we obtain

$$
-\sum_{i=1}^{N} p_{i} \log p_{i} \geqq-\sum_{i=1}^{N} \sum_{k=i}^{N} a_{k} / k \log 1 / k=\sum_{k=1}^{N} a_{k} \log k .
$$


Making the substitution $a_{k}=\theta_{k}-\theta_{k-1}$ (setting $\theta_{0}=0, \theta_{k}=a_{1}+a_{2}+\cdots+a_{k}$ ). we get

$$
-\sum_{i=1}^{N} p_{i} \log p_{i} \geqq \log N+\sum_{k=1}^{N-1} \theta_{k} \log k /(1+k) \geqq-\sum_{k=1}^{N} \theta_{k} / k+\log N .
$$

Now let $\nu(n)$ be a sequence of integers taken with the sole restriction that $\nu(n) \sim \varepsilon N(n)$ for some $\varepsilon>0$. Then from (1.34) we deduce: that

$$
\log N+\sum_{i=1}^{N} p_{i} \log p_{i} \leqq \theta_{\nu(n)} \log N+\log N / \nu(n) .
$$

Dividing by $\log N$ and passing to the limit we get

$$
\gamma_{0} \leqq \liminf _{n \rightarrow \infty} \theta_{\nu(n)} \text {. }
$$

In view of the fact that $p_{1}+p_{2}+\cdots+p_{\nu} \geqq a_{1}+a_{2}+\cdots+a_{\nu}$ and' the definition of $\nu(n)$ we deduce that the functions of $\left\{\overline{F_{n}}\right\}$ must all have a jump at the origin at least as big as $\gamma_{0}$. This establishes the inequality in (1.32).

Suppose we are given a sequence of probability distributions

$$
Q_{n}=\left\{q_{1}(n), q_{2}(n), \cdots, q_{N(n)}(n)\right\} .
$$

In case the $q_{i}(n)$ are not ordered we shall say that the sequence $\left\{Q_{n}\right\}$ is singular if and only if the ordered sequence $\left\{\mathfrak{P}_{n}\right\}$ that we obtain. by rearranging the probabilities of the $Q_{n}$ 's is a singular sequence. Similarly the deficiency of $Q_{n}$ will be the deficiency of $P_{n}$. Lemma 1.3. remains valid for unordered sequences of probability distributions.

\section{Proofs of the results.}

2.1. Theorem I.1 can be readily obtained from Theorem I.4. We shall thus concentrate in proving the latter. To this end we need the following result. Let $y$ and $y_{n}$ denote random variables. with distributions $F(x)$ and $F_{n}(x)$ respectively with $F(x)$ continuous. Assume in addition that the random variable $z_{n}=y-y_{n}$ is independent of $y_{n}$ and that $E\left(z_{n}^{2}\right) \rightarrow 0$.

LEMma 2.1. If $R_{n}$ is a sequence tending to zero in such a way that

$$
\lim \inf R_{n}^{2} / E\left(z_{n}^{2}\right)>0,
$$

then a necessary and sufficient condition for the distribution $F(x)$ to have a singular part is that for an $M$ so large that the quantity 


$$
\lambda_{n}=\sum_{\left|k R_{n}\right| \leqq M}\left[F_{n}\left(k R_{n}+R_{n}\right)-F_{n}\left(k R_{n}\right)\right]
$$

is bounded away from zero, the probability distributions

$$
Q_{n}=\left[\left(F_{n}\left(k R_{n}+R_{n}\right)-F_{n}\left(k R_{n}\right)\right) / \lambda_{n} ; \forall \text { integers } k \ni\left|k R_{n}\right| \leqq M\right]
$$

form a singular sequence.

This result follows from Lemma 2.5 of [3].

2.2. Assume then, with the notation and the hypotheses of Theorem I.4, that

$$
\liminf _{n \rightarrow \infty}\left(H_{1}+H_{2}+\cdots+H_{n}\right) / \log 1 / R_{n}<1 .
$$

Let us set

$$
y_{n}=x_{1}+x_{2}+\cdots+x_{n} .
$$

We shall visualize the probability space $\Omega$ where $y_{n}$ and $y$ are defined as the product of the probability spaces $\Omega_{1}, \Omega_{2}, \cdots, \Omega_{n}$ where the variables $x_{1}, x_{2}, \cdots, x_{n}$ are defined. Then the equivalence relation

$$
\omega^{\prime} \sim \omega^{\prime \prime} \text { if and only if } x_{n}\left(\omega^{\prime}\right)=x_{i}\left(\omega^{\prime \prime}\right) \text { for } i=1,2, \cdots, n
$$

generates a partition of $\Omega$ which is finer than the partition generated by the relation

$$
\omega^{\prime} \approx \omega^{\prime \prime} \text { if and only if } y_{n}\left(\omega^{\prime}\right)=y_{n}\left(\omega^{\prime \prime}\right) .
$$

Thus denoting by $D_{n}$ the entropy of the distribution of $y_{n}$, in view of well known properties of the entropy function, we shall have

$$
D_{n} \leqq H_{1}+\cdots,+H_{n} .
$$

Suppose now that $y_{n}$ takes the values

$$
y_{1 n}, y_{2 n}, \cdots, y_{N(n) n}
$$

with respective probabilities

$$
q_{1}(n), q_{2}(n), \cdots, q_{N(n)}(n) .
$$

We shall consider, for a given $M$, a partition of the indices $1,2, \cdots, N(n)$ into two sets $S^{\prime}$ and $S^{\prime \prime}$ defined as follows. $S^{\prime}$ is the set of all $i$ such that $\left|y_{i n}\right| \leqq M$ and $S^{\prime \prime}$ is the complement. Let

$$
\begin{array}{ll}
Q_{n}^{\prime}=\Sigma^{\prime} q_{i}, & D_{n}^{\prime}=-\Sigma^{\prime} q_{i} / Q_{n}^{\prime} \log q_{i} / Q_{n}^{\prime} \\
Q_{n}^{\prime \prime}=\Sigma^{\prime \prime} q_{i}, & D_{n}^{\prime \prime}=-\Sigma^{\prime \prime} q_{i} / Q_{n}^{\prime \prime} \log q_{i} / Q_{n}^{\prime \prime},
\end{array}
$$


where the summations $\Sigma^{\prime}$ and $\Sigma^{\prime \prime}$ are carried out over $S^{\prime}$ and $S^{\prime \prime}$ respectively. We have then that

$$
Q_{n}^{\prime} D_{n}^{\prime}+Q_{n}^{\prime \prime} D_{n}^{\prime \prime}=D_{n}+Q_{n}^{\prime} \log Q_{n}^{\prime}+Q_{n}^{\prime \prime} \log Q_{n}^{\prime \prime} \leqq D_{n} .
$$

By choosing $M$ sufficiently large, by the hypothesis I.8, we can guarantee that $Q_{n}^{\prime}$ after a while remains as close to one as we wish. In particular, since $D_{n}^{\prime \prime} \geqq 0$, by $2.23,2.24$ and the hypothesis 2.21 we can make sure that we have also

$$
\liminf _{n \rightarrow \infty} D_{n}^{\prime} / \log 1 / R_{n}<1 .
$$

In the subset of $\Omega$ where $\left|y_{n}\right| \leqq M$ we introduce a partition by means of the equivalence $\omega^{\prime} \cong \omega^{\prime \prime}$ if and only if $y_{n}\left(\omega^{\prime}\right)$ and $y_{n}\left(\omega^{\prime \prime}\right)$ belong to the same interval $\left[k R_{n}+R_{n}, k R_{n}\right)$. Since this partition is even coarser than the one induced by the equivalence in 2.22, for the entropy $E_{n}^{\prime}$ associated with this partition we must have

$$
E_{n}^{\prime} \leqq D_{n}^{\prime} \text {. }
$$

On the other hand if we let $\lambda_{n}$ be as in 2.11 and set

$$
E_{n}=-\sum_{\left|k R_{n}\right| \leqq M}\left[F_{n}\left(k R_{n}+R_{n}\right)-F_{n}\left(k R_{n}\right)\right] / \lambda_{n} \log \left[F_{n}\left(k R_{n}+R_{n}\right)-F_{n}\left(k R_{n}\right)\right] / \lambda_{n},
$$

we must have

$$
E_{n}^{\prime} \sim E_{n} .
$$

Combining this relation with (2.26) and (2.25) we deduce that

$$
\liminf _{n \rightarrow \infty} E_{n} / \log 1 / R_{n}<1 .
$$

Using this inequality in conjunction with Lemmas 1.3 and 2.1 we obtain the singularity of the distribution $F(x)$. This completes the proof of Theorem I.4

REMARK. It should be pointed out that Theorem I.4 gives a simpler condition and is more general than Theorem 2.6 of [3].

2.3. Proof of Theorem I.2. For a given $n$ and $p$ we can write the variable $y_{n p}$ defined in I.5 in the form

$$
y_{n p}(\omega)=y_{p}\left(\omega_{1}\right)+\beta^{p} y_{p}\left(\omega_{2}\right)+\cdots+\beta^{p(n-1)} y_{p}\left(\omega_{n}\right),
$$

where $y_{p}\left(\omega_{1}\right), y_{p}\left(\omega_{2}\right), \cdots, y_{p}\left(\omega_{n}\right)$ are supposed independent and equally distributed. If $m=n p+r$ where $0 \leqq r<p$ we have that

$$
y_{m}(\omega)=y_{n p}(\omega)+\beta^{n p} y_{r}\left(\omega_{n+1}\right) \text {. }
$$


By an argument similar to that used in $\S 2.2$ we deduce that the entropy of the variable $y_{m}(\omega)$ is less than or equal to the sum of the entropies of the summands. In other words, with the notation of the introduction, we must have

$$
H_{m}(\alpha) \leqq n H_{p}(\alpha)+H_{r}(\alpha) \text {. }
$$

Dividing by $m$ and passing to the limit as $m \rightarrow \infty$ we obtain

$$
\limsup _{m \rightarrow \infty} H_{m}(\alpha) / m \leqq H_{p}(\alpha) / p
$$

Since $p$ was arbitrarily chosen we deduce that

$$
\lim _{m \rightarrow \infty} H_{m}(\alpha) / m=\inf H_{p}(\alpha) / p .
$$

Clearly the limit in (2.31) may differ from $\log 2$ only when $\alpha$ satisfies polynomial equations with coefficients \pm 1 or 0 .

If $\alpha$ is a P. V. number it can be shown (for instance by means of Lemma 2.5 of [3]) that $H_{p}(\alpha) / p$ eventually takes values below $\log \alpha$. This accounts for the estimate in I.6. It would be of some interest to know whether or not I.6 holds for other than P. V. numbers.

2.4. Proof of Theorem I.3. For a P. V. number $\alpha$ we have the following estimates. If each $a_{i}(i=1,2, \cdots, n)$ takes only the values \pm 1 or 0 then either

$$
a_{1} \alpha+a_{2} \alpha^{2}+\cdots+a_{n} \alpha^{n}=0
$$

or

$$
\left|a_{1} \alpha+a_{2} \alpha^{2}+\cdots+a_{n} \alpha^{n}\right| \geqq c
$$

where $c$ is a constant depending only on $\alpha$. This result can be easily deduced from the definition of P. V. numbers. (See for instance Lemma 1.51 of [3]).

Let then $\left\{n_{k}\right\}$ be a sequence of constants satisfying the condition of Theorem I.3. If the numbers

$$
\pm \beta^{n_{1}} \pm \beta^{n_{2}} \pm \cdots \pm \beta^{n_{k}}
$$

(with $\beta=1 / \alpha$ ) were all distinct, the minimum distance between any two of them, in view of (2.41) would be greater than a fixed constant divided by $2^{k}$. Theorem 1.2 of [3] would then apply, and we would deduce that the distribution of the random variable

$$
y=\sum_{k=1}^{\infty} \pm \beta^{n_{k}}
$$


is absolutely continuous with a bounded derivative. This is of courseabsurd since it is known [2] that the distribution of

$$
\sum_{n=1}^{\infty} \pm \beta^{n}
$$

is singular.

2.5. We shall omit the proof of Theorem I.5, for it can be carried out step by step as it was done for Theorem 1.10 of [3]. The difference here is that Orlicz spaces methods would have to. replace the $L_{p}$ spaces methods used there. There is one point of the proof that is worth noting. Namely, it is known [1] that a bounded functional on an Orlicz space does not necessarily have an integral representation. However, at a point of our proof of Theorem 1.10 in [3] we use the Riesz representation theorem. Nevertheless, in carrying out the proof of Theorem I.5 even this point need not. be modified. In fact, for functionals of the type

$$
L(y)=\int_{-\infty}^{-\infty} y(x) d F(x)
$$

such a representation holds in an Orlicz space just as well as in an $L_{p}$ space.

\section{REFERENCES}

1. T. Andô, Linear functionals on Orlicz spaces, Nieuw Arch. Wisk. (3), 8 (1960), $1-16$.

2. P. Erdös, On a family of symmetric Bernoulli convolutions, Amer. J. Math. 61 (1939), 974-976.

3. A. Garsia, Arithmetic properties of Bernoulli convolutions Trans. Amer. Math. Soc.,. 102 (1962), 409-432.

4. B. Jessen and A. Wintner, Distribution functions and the Riemann zeta function, Trans. Amer. Math. Soc., 38 (1935), 48-88.

5. P. Levy, Séries don't les termes sont des variables éventuelles indépendantes Studia Math., 3 (1931), 119-155.

6. C. Pisot, La répartition modulo un et les nombres algebriques, Ann. Scuola Norm. Sup. Pisa, 2 (1938), 205-248.

7. C. Pisot and I. Dufresnoy, Sur un ensemble fermé de nombres algebriques, Ann.. Sci. Ecole Norm. Sup., 98 (1953), 105-133.

8. C. E. Shannon, The mathematical theory of comunication University of Illinois Press. (1949), Urbana Ill. 



\section{PACIFIC JOURNAL OF MATHEMATICS}

\section{EDITORS}

RalPh S. Phillips

Stanford University

Stanford, California

M. G. Arsove

University of Washington

Seattle 5 , Washington
J. Dugundu

University of Southern California

Los Angeles 7, California

Lowell J. Paige

University of California

Los Angeles 24, California

\section{ASSOCIATE EDITORS}
E. F. BECKENBACH
D. DERRY
H. L. ROYDEN
E. G. STRAUS
T. M. CHERRY
M. OHTSUKA
E. SPANIER
F. WOLF

\section{SUPPORTING INSTITUTIONS}

\author{
UNIVERSITY OF BRITISH COLUMBIA \\ CALIFORNIA INSTITUTE OF TECHNOLOGY \\ UNIVERSITY OF CALIFORNIA \\ MONTANA STATE UNIVERSITY \\ UNIVERSITY OF NEVADA \\ NEW MEXICO STATE UNIVERSITY \\ OREGON STATE UNIVERSITY \\ UNIVERSITY OF OREGON \\ OSAKA UNIVERSITY \\ UNIVERSITY OF SOUTHERN CALIFORNIA
}

\author{
STANFORD UNIVERSITY \\ UNIVERSITY OF TOKYO \\ UNIVERSITY OF UTAH \\ WASHINGTON STATE UNIVERSITY \\ UNIVERSITY OF WASHINGTON \\ AMERICAN MATHEMATICAL SOCIETY \\ CALIFORNIA RESEARCH CORPORATION \\ SPACE TECHNOLOGY LABORATORIES \\ NAVAL ORDNANCE TEST STATION
}

Mathematical papers intended for publication in the Pacific Journal of Mathematrcs should be typewritten (double spaced), and the author should keep a complete copy. Manuscripts may be sent to any one of the four editors. All other communications to the editors should be addressed to the managing editor, L. J. Paige at the University of California, Los Angeles 24, California.

50 reprints per author of each article are furnished free of charge; additional copses may be obtained at cost in multiples of 50 .

The Pacific Journal of Mathematics is published quarterly, in March, June, September, and December. Effective with Volume 13 the price per volume (4 numbers) is $\$ 18.00$; single issues, $\$ 5.00$. Special price for current issues to individual faculty members of supporting institutions and to individual members of the American Mathematical Society: $\$ 8.00$ per volume; single issues $\$ 2.50$. Back numbers are available.

Subscriptions, orders for back numbers, and changes of address should be sent to Pacific Journal of Mathematics, 103 Highland Boulevard, Berkeley 8, California.

Printed at Kokusai Bunken Insatsusha (International Academic Printing Co., Ltd.), No. 6 , 2-chome, Fujimi-cho, Chiyoda-ku, Tokyo, Japan.

\section{PUBLISHED BY PACIFIC JOURNAL OF MATHEMATICS, A NON-PROFIT CORPORATION}

The Supporting Institutions listed above contribute to the cost of publication of this Journal, but they are not owners or publishers and have no responsibility for its content or policies. 


\section{Pacific Journal of Mathematics}

\section{Vol. 13, No. $4 \quad$ June, 1963}

Dallas O. Banks, Bounds for eigenvalues and generalized convexity ........... 1031

Jerrold William Bebernes, A subfunction approach to a boundary value problem for

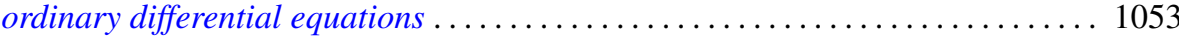

Woodrow Wilson Bledsoe and A. P. Morse, A topological measure construction . . . 1067

George Clements, Entropies of several sets of real valued functions . . . . . . . . . 1085

Sandra Barkdull Cleveland, Homomorphisms of non-commutative *-algebras . . . . . 1097

William John Andrew Culmer and William Ashton Harris, Convergent solutions of

ordinary linear homogeneous difference equations . . . . . . . . . . . . . . . 1111

Ralph DeMarr, Common fixed points for commuting contraction mappings . . . . . . 1139

James Robert Dorroh, Integral equations in normed abelian groups . . . . . . . . 1143

Adriano Mario Garsia, Entropy and singularity of infinite convolutions . . . . . . . 1159

J. J. Gergen, Francis G. Dressel and Wilbur Hallan Purcell, Jr., Convergence of extended Bernstein polynomials in the complex plane ................. 1171

Irving Leonard Glicksberg, A remark on analyticity of function algebras . . . . . . 1181

Charles John August Halberg, Jr., Semigroups of matrices defining linked operators

with different spectra ................................. 1187

Philip Hartman and Nelson Onuchic, On the asymptotic integration of ordinary

differential equations . . . . . . . . . . . . . . . . . . . . . . . . . . . . 1193

Isidore Heller, On a class of equivalent systems of linear inequalities . . . . . . . . . 1209

Joseph Hersch, The method of interior parallels applied to polygonal or multiply

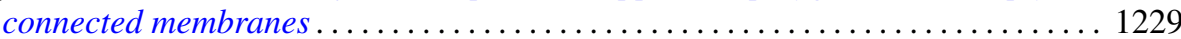

Hans F. Weinberger, An effectless cutting of a vibrating membrane . . . . . . . . . . 1239

Melvin F. Janowitz, Quantifiers and orthomodular lattices ....

Samuel Karlin and Albert Boris J. Novikoff, Generalized convex inequalities . .

Tilla Weinstein, Another conformal structure on immersed surfaces of negative

curvature.

Gregers Louis Krabbe, Spectral permanence of scalar operators

Shige Toshi Kuroda, Finite-dimensional perturbation and a representaion of

scattering operator.

Marvin David Marcus and Afton Herbert Cayford, Equality in certain

inequalities

Joseph Martin, A note on uncountably many disks .

Eugene Kay McLachlan, Extremal elements of the convex cone of semi-norms . . . . 1335

John W. Moon, An extension of Landau's theorem on tournaments . .

Louis Joel Mordell, On the integer solutions of $y(y+1)=x(x$

Kenneth Roy Mount, Some remarks on Fitting's invariants .....

Miroslav Novotný, Über Abbildungen von Mengen ............

Robert Dean Ryan, Conjugate functions in Orlicz spaces.

John Vincent Ryff, On the representation of doubly stochastic operators . . . . . . . . 1379

Donald Ray Sherbert, Banach algebras of Lipschitz functions .

James McLean Sloss, Reflection of biharmonic functions across analytic boundary

conditions with examples.

L. Bruce Treybig, Concerning homogeneity in totally ordered, connected topological space....

John Wermer, The space of real parts of a function algebra...

James Juei-Chin Yeh, Orthogonal developments of functionals and related theorems

in the Wiener space of functions of two variables......... 